\title{
Les nouvelles technologies
}

\section{de l'information et}

\section{de la communication : quel impact auprès de nos étudiants?}

D ans cette livraison du journal, JD Ricard et coll. soulèvent une question fondamentale mais malheureusement trop peu souvent posée : " quelle est I'utilisation réelle des outils de formation mis à la disposition de nos étudiants? ».

Leur articleintitulé «Utilisation desnouvelles technologies de l'information et de la communication par les étudiants en médecine. Enquête de pratique et mise en situation ${ }^{1}$, qui rend compte d'une enquête effectuée auprès de leurs étudiants, apporte à cet égard une réponse sans doute provisoire, mais en tout état de cause sans appel : bien qu'ayant un accès Internet à leur disposition, la majorité des étudiants n'utilisent que très peu les outils didactiques et les ressources éducatives disponibles en ligne! Quatrevingt dix huit pour cent des étudiants interrogés ne connaissent pasI'U niversité médicale virtuell e francophone et utilisent plus fréquemment des sites créés par l'industrie pharmaceutique ou des sites plus institutionnels.

Certes, l'échantillon est restreint puisqu'il ne concerne que 50 étudiants provenant d'une seule faculté. Ces résultats concordent néanmoins avec ceux de Reding et coll. ${ }^{2}$, qui montraient en 2001 qu'une majorité d'étudiants sestimaient insuffisamment formés à l'usage des nouvelles technologies de l'information et de la communication (NTIC) et privilé giaient l'assimilation de la matière via des polycopiés. Le constat en 2005 semble reproduire celui de 2001 : les N TIC n'ont pas réalisé la percée... que promettaient les concepteurs.

U ne courte revue de la littérature publiée il y a deux ans ${ }^{3}$ montrait que:

1) les véritables potentiels des NTIC pour l'éducation ne peuvent se limiter à une approchetechnologique: I'ordinateur superposé à des formes traditionnelles d'enseignement n'améliore ni la qualité, ni le rendement de l'enseignement ;

2) les bénéfices attendus de I'utilisation des NTIC ne doivent pas se limiter à la seule sphère du savoir. L'introduction de nouvelles techniques suppose une restructuration en profondeur des méthodes didactiques;

3) insérer de nouvelles technologies n'induit 
pas automatiquement l'introduction de nouvelles méthodes d'enseignement.

Avec M . Lebrun ${ }^{4}$, nous pensonsqueles N TIC doivent pouvoir influencer les étudiants dans cinq champs:

1) leur motivation à travailler de manière individuele;

2) la qualité de l'information mise à leur dis position via Internet;

3) la richesse et la variété des travaux personnels qui leur sont demandés;

4) I'importance des interactions que les N T IC peuvent permettre, à la fois entre eux ou avec leurs enseignants; 5) la facilitation de leur capacité d'expression.

Dans leurs conclusions, JD Ricard et coll. mettent en exergue la déficience de l'information concernant les sites mis à disposition des étudiants. Cela est certainement vrai. M ais, au-delà de ce constat purement factuel, il convient de rappeler plus que jamais l'impé rieuse exigence que la réflexion sur les principes et le « design » pédagogiques précède l'implantation technologique et non l'inverse.

Jean-François $D$ enef mailto : denef@isto.ud.ac.be

\section{Réérérences}

1. RicardJ-D, Lejoyeux M, El-Ghoneimi A, M atheron $S, M$ aillard $D$, Crickx $B$, D reyfuss $D$ \& coll. Utilisation des nouvelles technologies de l'information et de la communication par les éudiants en médecine Enquête de pratique et mise en situation. Pédagogie M édicale 2005 ; 6 : 112-122.

2. Reding R, D ene J J, Parmentier P, Lebrun M . Accès, compétence et opinions des étudiants visà vis des technologies de l'information et la communication. Pédagogie médicale $2001 ; 2$ : 242-249.
3. D enef JF, Lebrun $M, D$ onckelsF. Téééformation, téé médecine, $\mathrm{E}-\ldots$. M ythe ou réalité? Louvain M édical $2003 ; 122:$ S335-S342.

4. Lebrun M . Théories et méthodes pédagogiques pour enseigner et apprendre. Quelle place pour les TIC dansl'éducation ? BruxellesParis: De Boeck, 2002. 